\title{
Modified Bipartite Matching for Multiobjective Optimization: Application to Antenna Assignments in MIMO Systems
}

\author{
Fanglei Sun, Victor O. K. Li, Fellow, IEEE, and Zhifeng Diao
}

\begin{abstract}
Based on the Hungarian algorithm, the KuhnMunkres algorithm can provide the maximum weight bipartite matching for assignment problems. However, it can only solve the single objective optimization problem. In this paper, we formulate the multi-objective optimization (MO) problem for bipartite matching, and propose a modified bipartite matching (MBM) algorithm to approach the Pareto set with a low computational complexity and to dynamically select proper solutions with given constraints among the reduced matching set. In addition, our MBM algorithm is extended to the case of asymmetric bipartite graphs. Finally, we illustrate the application of MBM to antenna assignments in wireless multiple-input multiple-output (MIMO) systems for both symmetric and asymmetric scenarios, where we consider the multi-objective optimization problem with the maximization of the system capacity, total traffic priority, and long-term fairness among all mobile users. The simulation results show that MBM can effectively reduce the matching set and dynamically provide the optimized performance with different quality of service $(\mathrm{QoS})$ requirements.
\end{abstract}

Index Terms-Multi-objective optimization, bipartite matching, resource allocation.

\section{INTRODUCTION}

$\mathbf{T}$ HE Kuhn-Munkres method for the assignment problem describes an algorithm for constructing a maximum weight perfect matching in a bipartite graph [1] [2]. It is proved that the Kuhn-Munkres algorithm can always find the maximum assignment, i.e., an optimal solution to the maximum weight sum [2]. Compared with exhaustive search, the computational complexity is reduced from $O(n !)$ to $O\left(n^{3}\right)$, where $n$ is the cardinality of the node set of a symmetric bipartite graph. However, the Kuhn-Munkres method only considers single objective optimization, and is not applicable to multi-objective optimization. In addition, for asymmetric bipartite graphs, the Kuhn-Munkres algorithm only considers the assignments based on the minimum cardinality of the bipartite node sets. It will cause unnecessary resource wastage when the redundant resource is reusable.

Manuscript received August 18, 2007; revised December 1, 2007 and March 27, 2008; accepted May 12, 2008. The associate editor coordinating the review of this paper and approving it for publication was J. Luo. This research is supported in part by the Research Grants Council of the Hong Kong Special Administrative Region, China, under Grant No. HKU 7148/06E.

F. Sun and Victor O. K. Li are with the Department of Electrical and Electronic Engineering, The University of Hong Kong, Hong Kong, China (e-mail: \{flsun,vli\}@eee.hku.hk).

Z. Diao is with Huawei Technologies CO., LTD, Shanghai, China (e-mail: zfdiao@huawei.com).

Digital Object Identifier 10.1109/TWC.2009.071351
In network design, a single global objective, which may be one of the network performance measures, such as throughput, is often examined to optimize network performance. This approach is meaningful in the homogeneous situation, such as when each user has the same quality of service (QoS) requirement. In this case, equal performance is the implied fairness criterion, and global optimality is equivalent to individual optimization. However, in the heterogeneous case, individual requirements may be ignored by using a single overall objective, and sometimes users may have to sacrifice their individual performance, such as priority, delay or fairness, for the goodness of the entire network [3]-[5].

In this paper, we take wireless multiple-input multipleoutput (MIMO) communication systems with spatial multiplexing as an example. Combined with multiuser diversity [6] in cellular packet transmission systems, a couple of antenna assignment schemes have been proposed for packet schedulers to maximize the system capacity or to provide fairness among mobile users [7]-[9]. However these scheduling schemes only consider single objective optimization. None of them considers multi-objective optimization which accounts for the maximization of capacity, total priority, long-term fairness, and so on.

In our proposed modified bipartite matching (MBM) algorithm, a key objective is chosen from all objectives. For this objective, the Kuhn-Munkres algorithm is applied to each updated bipartite graph obtained by deleting one edge at a time till no edge remains in the graph, and MBM chooses the matching which approaches the optimal solution or satisfies the system requirements. To decide which edge should be deleted, we need to identify the edge which has the least contribution to the other sub-key optimization objectives individually. If the edges corresponding to the least contribution in each of the different sub-key optimization objectives are the same, then delete this edge in the bipartite graph. But in general, these edges are different. Then we compare the values of the key objective if these edges are deleted individually, and delete the edge which is deduced to have the least contribution to the optimization of the key objective. For asymmetric bipartite graphs, i.e. the number of nodes in the two bipartite sets are different, if the resource to be assigned may be fully utilized, our MBM is designed to maximize the resource usage by enhancing the graph and finding updated matchings. As far as we know, this paper is the first work to address the multi-objective optimization problem in the bipartite matching field and MBM is the first algorithm proposed for solving this 
problem.

The paper is organized as follows. Section II presents the formulation of the MO problem for bipartite matchings. The details of the MBM algorithm is described in Section III. In Section IV, an example of MBM for antenna assignments in wireless MIMO communication systems is given. Section V concludes this paper.

\section{Formulation of MO Problem for Bipartite MATCHINGS}

\section{A. Multiobjective Optimization (MO)}

For most multi-objective optimization problems, there exists a set of non-dominated solutions that have a trade-off relationship with each other, and one of the multiple objectives of each solution cannot be improved without sacrificing the others [10]. This concept is known as the Pareto optimality [11].

Definition: Consider, without loss of generality, the maximization of the $n$ components $f_{k}, k=1, \ldots, n$, of a vector function $\boldsymbol{f}$ of a vector variable $\boldsymbol{x}$ in a universe $\mathbf{X}$, where

$$
\boldsymbol{f}(\boldsymbol{x})=\left(f_{1}(\boldsymbol{x}), \ldots, f_{n}(\boldsymbol{x})\right) \text {. }
$$

Then a decision vector $\boldsymbol{x}_{u} \in \mathbf{X}$ is said to be Pareto optimal if and only if there is no $\boldsymbol{x}_{v}$ for which $\boldsymbol{v}=\boldsymbol{f}\left(\boldsymbol{x}_{v}\right)=$ $\left(v_{1}, \ldots, v_{n}\right)$ dominates $\boldsymbol{u}=\boldsymbol{f}\left(\boldsymbol{x}_{u}\right)=\left(u_{1}, \ldots, u_{n}\right)$, that is, there is no $\boldsymbol{x}_{v}$ such that $v_{i} \geq u_{i}, \forall i \in\{1, \ldots, n\}$ and $v_{i}>$ $u_{i}, \exists i \in\{1, \ldots, n\}$.

The set of all Pareto-optimal decision vectors is called the Pareto optimal set or the Pareto frontier of the problem. The corresponding set of objective vectors is called the nondominated set. In practice, however, it is not unusual for these two terms to be used interchangeably to describe solutions of a multi-objective optimization problem. The notion of Pareto optimality is only the first step towards the practical solution of a multi-objective problem, which usually involves the choice of a single compromise solution from the non-dominated set according to some preference information. Therefore, the first task of multi-objective optimization is to find the Pareto set, from which a proper solution is selected in the second step.

Based on the Pareto principle, the algorithm NSGA-II (Nondominated Sorting in Genetic Algorithm) proposed by Deb in [12] and the Jensen's algorithm [13] are the two key methods to get the Pareto set. Their computational complexities are $O\left(r N^{2}\right)$ and $O\left(N \log ^{(r-1)} N\right)$, where $r$ is the number of objectives and $N$ is the population size. The pseudo-code of NSGA-II can be found in [12].

\section{B. MO Problem for Bipartite Matchings}

A graph is denoted by $G(V, E)$, where $V$ is the vertex set and $E$ is the edge set of the graph. If $V=X \cup Y$ with $X \cap Y=\Phi$, and each edge in $E$ has one endpoint in $X$ and the other in $Y$, the graph $G(V, E)$ is a bipartite graph, which can also be denoted as $G(X, Y, E)$. The bipartite graph is very useful for some applications, such as an assignment problem which can be depicted as follows: Given a weighted complete bipartite graph $G=(X, Y, E)$, find a matching $m$ from $X$ to $Y$ with the maximum sum of weights, where the cardinality of $X$ and $Y$ are $n_{1}$ and $n_{2}$, each $x$ in $X$ is connected to $n_{2}$ vertices in $Y$ and edge $(x, y)$ has weight $w(x, y)$. A matching is a set of edges such that no two edges have a common vertex. In a job assignment application, $X$ could be a set of workers, $Y$ a set of jobs, and $w(x, y)$ is the earnings made by assigning worker $x$ to job $y$. The essence of the assignment problem is to find the optimal matching. Traditionally, bipartite matching problems only focus on a single objective. However in many cases, the system may have multiple objectives to optimize. To solve this problem, we firstly formulate the MO problem for bipartite matchings. Considering a symmetric bipartite graph (asymmetric cases will be discussed in the next section), by exhaustive searching, there are $n$ ! matchings, denoted as $\left\{m_{1}, m_{2}, \ldots, m_{n !}\right\} \in M$, where $n$ is the cardinality of $X$ or $Y$ and $M$ is the set of all possible matchings. By the definition of $\mathrm{MO}$ in the above subsection, assuming there are $l$ optimization objectives, namely $f_{i}(m), i=1, \ldots, l$, the MO problem for the bipartite matching is formulated as the maximization of the $l$ components $f_{i}, i=1, \ldots, l$, of a vector function $f$ of a vector variable $m$ in $M$, where

$$
\boldsymbol{f}(m)=\left(f_{1}(m), \ldots, f_{l}(m)\right) .
$$

For the functions $f_{i}(m), i=1, \ldots, l$, we further classify them into two types according to the application requirements.

(1) Type 1: the variation of $f_{i}^{1}(m)$, denoted as $\triangle f_{i}^{1}(m)$, only depends on the changed edge weights, such as a sum-performance criterion. As mentioned before, the weight $w(x, y)$ may be the earnings, time, or efficiency to assign worker $x$ to job $y$.

(2) Type 2: the variation of $f_{i}^{2}(m)$ not only depends on the changed edge weights, but also on the unchanged ones when the matching $m$ is updated to $m^{\prime}$, such as a fairness index proposed in [14].

\section{MBM ALGORITHM}

\section{A. Symmetric Bipartite Matching}

With the single objective optimization, it has been proved that the Kuhn-Munkres algorithm can always find the maximum weight matching for a bipartite graph. The KuhnMunkres algorithm is based on the procedure of the Hungarian algorithm. The details of these two algorithms can be found in [8] and [15].

Among multiple objectives, MBM firstly choose a key objective, and the others are the sub-key objectives. In this section, based on the job assignment system, MBM is illustrated with three objectives to be maximized, which are the earnings, working efficiency and earning fairness for all workers, denoted as $f_{1}\left(m_{i}\right), f_{2}\left(m_{i}\right)$, and $f_{3}\left(m_{i}\right), m_{i} \in M$. Let $w(x, y)$ and $s(x, y)$ be the weights associated with the earnings and working efficiency. These two objectives are to maximize the sum of all weights, so they belong to Type 1 objectives. However, the fairness evaluation is based on the more complicated computation of weights $w(x, y)$, and it is a Type 2 objective. Among these three objectives, the earnings objective is set as the key objective. Under exhaustive enumeration, the number of elements in $M$ is $n$ !, where $n$ is the total number of workers. To reduce the size of the 
matching set $M$, MBM tries to update the bipartite graph $G$ by deleting an edge in each iteration. In each iteration, MBM firstly applies the Kuhn-Munkres algorithm for the key objective maximization for the updated bipartite graph $G^{\prime}$, whose initial state is $G$. Then a perfect matching $m$ in this iteration is achieved, and $w\left(x_{j}, y_{j}\right), j=1, \ldots, n$, are the weights of the edges in this matching $m$. The key idea of MBM is to delete an edge at a time among the edges in $m$, namely setting the corresponding weight to zero, to balance the performance of the key and sub-key objectives. The deleting operation is repeated until no edges are left in the bipartite graph $G^{\prime}$. After each implementation of the KuhnMunkres algorithm, the perfect matching is collected to the matching set $M$, and its corresponding performance results of all optimization objectives are recorded for later solution comparisons. Since for a complete bipartite graph, there are edges between each pair of nodes in the two separated node sets, the number of all edges is $n^{2}$, the upper bound of the number of iterations is $O\left(n^{2}\right)$, that is the maximum size of the matching set $M$ is reduced to $n^{2}$. In each iteration, a perfect matching $m$ with $n$ edges is obtained by the Kuhn-Munkres algorithm. The remaining issue to be addressed is to decide which edge to delete in this matching $m$. In MBM, since the Kuhn-Munkres algorithm is applied to optimize the key objective, for the edge deleting operation in $m$, we consider the performance of the sub-key objectives. The detailed processing is shown as follows:

(1) For each sub-key objective, choose the edge which has the least contribution to the optimization of this objective. For the two types of objectives we have different evaluation schemes:

i) For Type 1 objectives: Since the values of these objectives are obtained as simple summation of the given weights, we can easily update the values of $f_{i}\left(m^{\prime}\right)$ by comparing the changed weights, where $m^{\prime}$ is the matching without the edge to be deleted. Obviously, the corresponding deleted edge is the edge which has the least weight contribution for this objective.

ii) For Type 2 objectives: The variation of $f_{i}\left(m^{\prime}\right)$ can not be easily obtained only by the changed weights. To simplify the computation, in MBM, we try to find some simple ways to decide which edges have the least contribution to these objectives. For example, suppose we consider the earning fairness. For a given matching $m$ with weights $w_{j}, j=1, \ldots, n$, MBM calculates $\eta_{j}$ of the different edges by

$$
\eta_{j}=\left|w_{j}-\left(\sum_{i=1}^{n} w_{i}\right) / n\right|^{2}, \quad j=1, \ldots, n .
$$

Then we choose the edge $q$ as the edge which has the least contribution to the system fairness performance by

$$
\{q\}=\operatorname{argmax}_{j}\left\{\eta_{j}\right\}, \quad j=1, \ldots, n .
$$

After choosing the edges which have least contribution for the sub-key objectives, if these edges are identical, the final edge to be deleted for this iteration is found. Otherwise, perform the following processing.

(2) Generally speaking, edges which have the least contribution to the performance of different sub-key objectives are not identical. For these cases, MBM will resort to the key
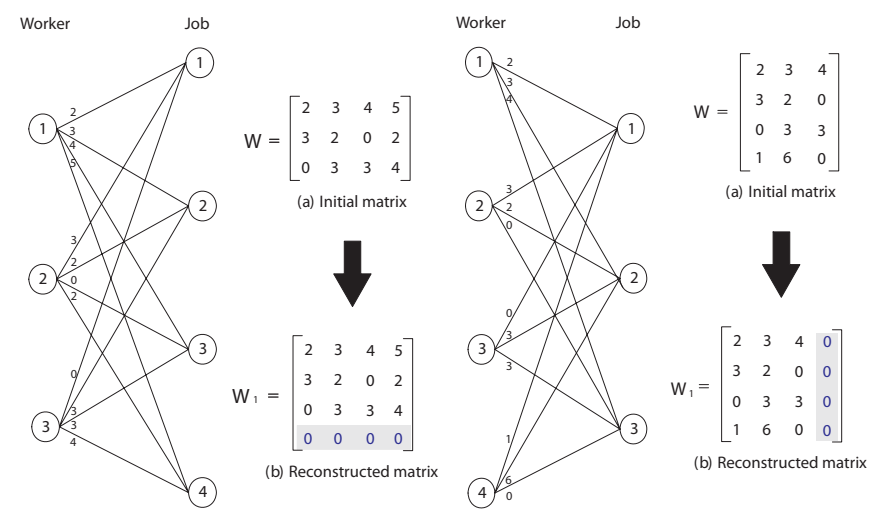

Fig. 1. Asymmetric bipartite matching (1).

objective to make the final deletion decision. It will identify the edge with the least contribution to the key objective and delete it.

MBM try to gradually increase the performance of the subkey objectives, while maximizing the performance of the key objective for each updated $G^{\prime}$. Note that since there is no backtracking in our algorithm to allow us to add back previously deleted edges, our solution does not guarantee strict monotone decrease of the maximum weighted matching. However, in our test cases, the loss of performance of the key objective improves the performance of the sub-key objectives, thus balancing the performance of the whole system.

For a general MO problem, current solutions, such as NSGA-II, focus on giving the Pareto set or the optimal set as solutions. However, for an MO problem which requires a specific solution, as we mentioned in Section II-A, even given the Pareto set, how to select a proper solution from the found Pareto set is still a problem. In this work, enlightened by $\varepsilon$-constraint method in [16] [17], we also transform an MO problem to a single objective optimization problem by changing the $(l-1)$ sub-key objectives as the constraints of the key objective. By setting different constraints, this method can be used to get an optimal set. However in our work, it is used to get a specific solution at the second step in MBM. The problem is further modified to the following formulation:

$$
\left\{\begin{array}{c} 
\\
\text { s.t. } \quad f_{2}(m) \leq \varepsilon_{1}, \\
\cdots \cdots \\
\\
f_{l}(m) \leq \varepsilon_{(l-1)}, \quad m \in M,
\end{array}\right.
$$

where $\varepsilon_{i}(i=1, \ldots,(l-1))$ are the parameters given by the system to represent the optimization targets of the sub-key objectives. With these constraints, MBM will find a proper matching from our reduced matching set $M$, such that these constraints are met as much as possible.

\section{B. Asymmetric Bipartite Matching}

In MBM, our key idea is to enhance an asymmetric graph to a symmetric one, and then deal with the MO problem by the same processing introduced for symmetric bipartite matchings. Firstly, in the case when $n_{1}<n_{2}$, as shown in 


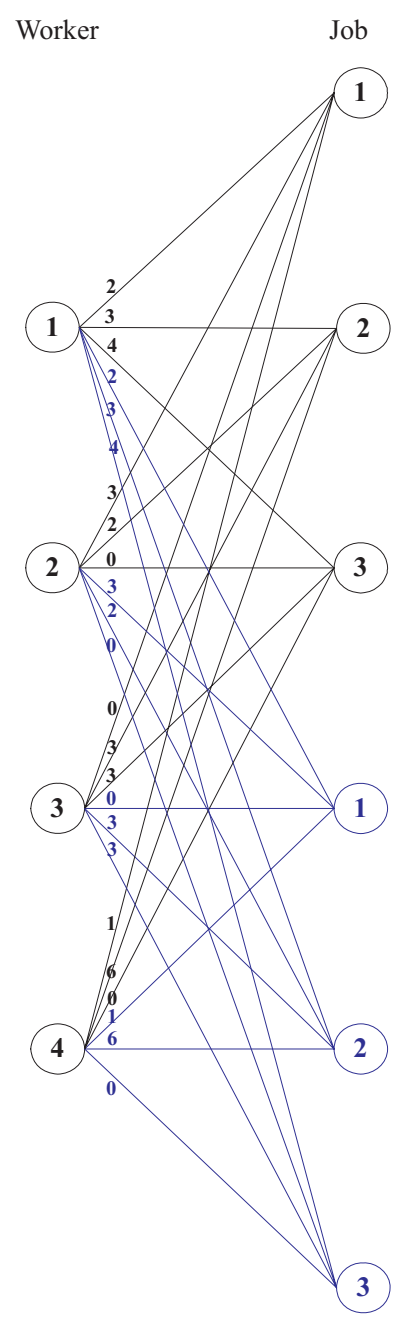

$\mathbf{W}_{1}=\left[\begin{array}{llllll}2 & 3 & 4 & 2 & 3 & 4 \\ 3 & 2 & 0 & 3 & 2 & 0 \\ 0 & 3 & 3 & 0 & 3 & 3 \\ 1 & 6 & 0 & 1 & 6 & 0\end{array}\right]$

(b) Copy the nodes
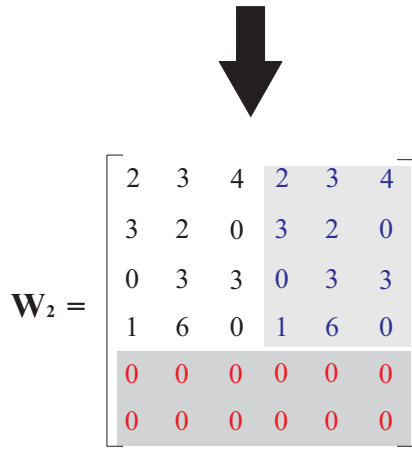

(c) Reconstruct the matrix

Fig. 2. Asymmetric bipartite matching (2).

the left subfigure of Fig. 1, our MBM appends $\left(n_{2}-n_{1}\right)$ allzero rows to the original weight matrix $W_{n_{1} \times n_{2}}$ to form a square weight matrix $W_{n_{2} \times n_{2}}$..

Secondly, when $n_{1}>n_{2}$, if the redundant worker resource is ignored, as presented above, MBM algorithm will append $\left(n_{1}-n_{2}\right)$ all-zeros columns to construct a square weight matrix, as shown in the right subfigure of Fig. 1. However, this method will cause wastage of worker resource. If the redundant worker resource is reusable, and the could help other workers to finish the jobs, then the working efficiency of the system is improved by the processing in Fig. 2.

\section{Analysis of the Computational Complexities}

In this subsection we compare the computational complexities of the exhaustive search, Pareto set solution, and our proposed MBM algorithms. A summary of the comparison is shown in Table I.

For single objective optimization, assuming the weight matrix of a bipartite graph is $W_{n_{1} \times n_{2}}$, the computational complexity of the Hungarian algorithm is $O(n|E|)$, where $|E|$ is the number of edges in $G$, and $n$ is the cardinality of the vertex set of the symmetric bipartite graph. The computational
TABLE I

Computational Complexities

\begin{tabular}{|c|c|}
\hline Assignment scheme & Computational complexity \\
\hline \hline Exhaustive search (Whole matching set) & $O\left((r+1)\left(\frac{t !}{(t-s) !}\right)\right)$ \\
\hline NSGA-II (Pareto set) & $O\left(r\left(\left(\frac{t !}{(t-s) !}\right)^{2}+N_{\text {Pareto }}\right)\right)$ \\
\hline MBM (Reduced optimal set) & $O\left(n_{1} n_{2}\left(t^{3}+r(s+1)\right)\right)$ \\
\hline
\end{tabular}

complexity of the Kuhn-Munkres algorithm is $O\left(t^{3}\right)$ [2], where $t=\max \left(n_{1}, n_{2}\right)$. For multi-objective optimization problems, the general algorithms are divided into two steps: the first step is to obtain an optimal set, and the second step is to select a proper solution from it. For the first step, we compared the computational complexities of exhaustive search, Pareto set solution and our MBM algorithm to get different optimal sets.

It is obvious that the computational complexity of the exhaustive search to obtain the whole matching set is

$$
O\left(C_{t}^{s} P_{s}\right)=O\left(\frac{t !}{(t-s) !}\right)
$$

where $t=\max \left(n_{1}, n_{2}\right)$ and $s=\min \left(n_{1}, n_{2}\right) . C_{t}^{s}$ is the number of ways of selecting a subset of $s$ elements from a set of $t$ elements. It is an un-ordered collection of unique sizes. $P_{s}$ is the permutation of a set of $s$ elements. A permutation is an ordered sequence of elements selected from a given finite set, without repetitions. If the NSGA-II algorithm [12] is considered, the computational complexity to obtain the Pareto set is $O\left(r N^{2}\right)$, where $r$ is the number of objectives and $N$ is the population size. As deduced before, the size of the whole matching set is

$$
N_{\text {Exhaustive search }}=\frac{t !}{(t-s) !} .
$$

Therefore, the total computational complexity to obtain the Pareto set is $O\left(r\left(\frac{t !}{(t-s) !}\right)^{2}\right)$. However for our MBM search, since there are at most $n_{1} \times n_{2}$ edges in $G$, by the edge deletion process, the size of the matching set could be reduced to

$$
N_{M B M}=n_{1} \times n_{2} .
$$

In addition, for each iteration, the Kuhn-Munkres algorithm is applied to obtain the perfect matching of the updated bipartite graph and the computational complexity of the deletion operation is $O(r s)$, so the computational complexity of MBM at the first step to obtain our optimal set is $O\left(t^{3}+r s\right)$.

For the second steps of different algorithms, we assume the same $\varepsilon$-constraint method is adopted to pick one objective as the key objective; the other objectives are used as constraints of the key objective, as shown in (3). The computational complexity at the second stage depends on the size of the solution set obtained in the first stage. If the cardinality of the solution set is $N$, the computational complexity of the second step is $O(r N)$. Therefore, the total computational complexity of the exhaustive search is $O\left((r+1)\left(\frac{t !}{(t-s) !}\right)\right)$, and that of MBM is $O\left(n_{1} n_{2}\left(t^{3}+r(s+1)\right)\right)$. However, for the Pareto set method, the cardinality of the Pareto set is dynamically changed in different cases, and we assume it is 


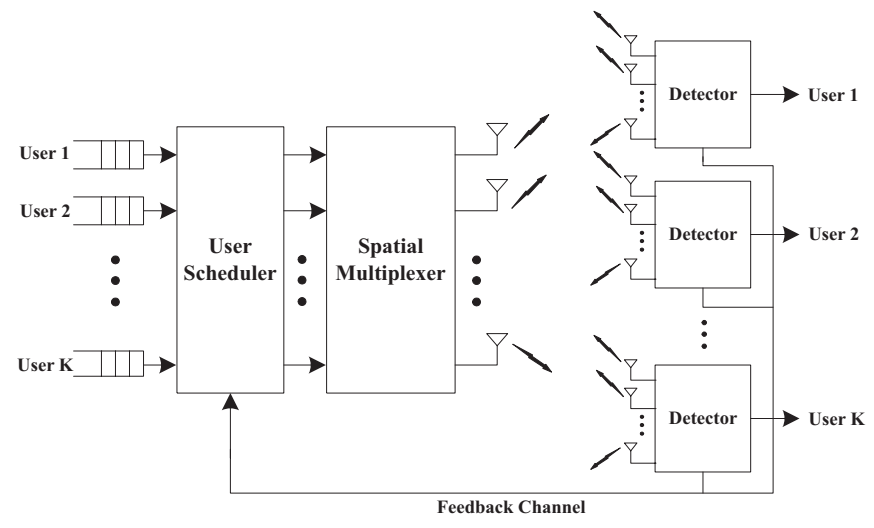

Fig. 3. Downlink MIMO system.

$N_{\text {Pareto }}$ which is generally between $n_{1} n_{2}$ and $\frac{t !}{(t-s) !}$, so the total computational complexity is $O\left(r\left(\left(\frac{t !}{(t-s) !}\right)^{2}+N_{\text {Pareto }}\right)\right)$.

\section{EXAMPLE}

\section{A. System Model}

We illustrate the application of MBM to an antenna assignment problem. The system model is shown in Fig. 3, where the base station has $N_{T}$ antennas and each mobile user has $N_{R}\left(\geq N_{T}\right)$ antennas. The number of mobile users is $K$. The transmit power is equally divided among the transmit antennas. Based on the post-detection signal-to-noise ratio (SNR), the scheduler selects a group of users and assigns the antennas to them to transmit in the time-slot. The channel matrix $H_{k}(t)$ between the base station and user $k$ at time-slot $t$ is [6]

$$
H_{k}(t)=\sqrt{S N R_{0} \cdot\left(l_{k} / L\right)^{-\beta} \cdot 10^{S_{k} / 10}} \cdot G_{k}(t),
$$

where $S N R_{0}$ is the median $S N R, L$ is the cell radius, $G_{k}(t)$ is an independent complex Gaussian random variable with zero mean and unit variance, $\beta$ is the path loss exponent, and $S_{k}(t)$ is a real Gaussian random variable with zero mean and variance $\sigma_{S}^{2}$. In addition, $l_{k}$ is the distance between the base station and user $k$. The distance of each user from the base station is uniformly distributed between zero and $L$. The postdetection SNR [8] is defined as the SNR of a transmit symbol after minimum mean-squared error (MMSE) detection, and the corresponding value for the transmit antenna $n$ to user $k$ can be calculated as (8).

In (8), $P_{R}$ is the total received signal power and $\sigma_{N}^{2}$ is the noise power per receive antenna. The weight matrix for MMSE is given as

$$
W_{k}(t)=H_{k}^{H}(t)\left(H_{k}(t) H_{k}^{H}(t)+\left(\sigma_{N}^{2} N_{T} / P_{R}\right) I_{N_{R}}\right)^{-1},
$$

where $(\cdot)^{H}$ denotes the conjugate transpose and $I_{N_{R}}$ is the $N_{R} \times N_{R}$ identity matrix. Therefore, the system capacity for time slot $t$ can be expressed as

$$
C(t)=\sum_{n=1}^{N_{T}} \log _{2}\left(1+\gamma_{k, n}(t)\right)
$$

\section{B. Problem Formulation}

In spatial multiplexing, multiple streams are transmitted through different transmit antennas. To exploit multiuser diversity, the scheduler can simultaneously choose as many multiple users as transmit antennas and allocate each user one or more antennas. In this wireless MIMO system, we consider the MO problem with the following three objectives to be optimized:

(1) Capacity: We denote by $C\left(k_{1}, \ldots, k_{N_{T}}\right)$ the capacity achieved by allocating transmit antenna $i$ to user $k_{i}$ for $i=$ $1, \ldots, N_{T}$. Consequently, our proposed MBM aims to search $k_{1}, \ldots, k_{N_{T}}, \forall i, j(\neq i) ; k_{i} \neq k_{j}$ to

$$
\max C\left(k_{1}, \ldots, k_{N_{T}}\right)=\max \sum_{i=1}^{N_{T}} \log _{2}\left(1+\gamma_{k_{i}, i}\right) .
$$

(2) Long-term fairness: In a wireless communication system, the fairness index is widely used to evaluate the fairness performance [18]. It takes values between 0 and 1 . In the simulations, we consider the long-term fairness performance and define the corresponding fairness index as follows:

$$
f=\frac{\left(\sum_{i=1}^{N_{T}} \overline{C_{k_{i}, i}}\right)^{2}}{N_{T}\left(\sum_{i=1}^{N_{T}}{\overline{C_{k_{i}, i}}}^{2}\right)},
$$

where $\overline{C_{k_{i}, i}}$ is the average transmit rate achieved in the past slots. The larger the long-term fairness index, the better the system fairness performance. So our MBM also tries to maximize the long-term fairness index.

(3) Priority: To support the QoS for multimedia transmissions, we assume there are $P_{t}$ types of traffics supported in a MIMO system. In addition, we further classify the users' service priorities into $P_{u}$ levels, for example by their service charge. The user who pays more for service will enjoy higher transmission priority. In our simulations, we set both $P_{t}$ and $P_{u}$ to 8 . We assume all types of traffic flows for different users arrive randomly, so we randomly generate the priority weights $s_{k, n}$ from antenna $n$ to user $k$ with a random integer in the range $\left[1, \ldots, P_{t} \times P_{u}\right]$. Then the optimization objective is to meet more transmission requirements which enjoy higher service priorities, namely,

$$
\max \sum_{i=1}^{N_{T}} s_{k, n}, \quad s_{k, n} \in\left[1, \ldots, P_{t} \times P_{u}\right] .
$$

Among these three objectives, capacity is selected as the key objective, while fairness and priority are considered as sub-key objectives.

\section{Simulation Results}

The above three objectives for optimizing MIMO systems are now evaluated through simulations, for both symmetric and asymmetric bipartite matching. We assume $S N R_{0}=10 \mathrm{~dB}$, the path loss exponent $\beta=3.7 \mathrm{~dB}$, the log standard deviation of shadow fading $\sigma_{S}=8 \mathrm{~dB}$, the cell radius $L=1 \mathrm{Km}$, and $P_{R} / \sigma_{N}^{2}=10 \mathrm{~dB}$. In this model, the distance of the four users from the base station is uniformly distributed between 0 and $L$. Simulation time is $2 \times 10^{3}$ time slots (Ts). Our simulation tool is Matlab V7.01. 


$$
\gamma_{k, n}(t)=\frac{\left|\left[W_{k}(t) H_{k}(t)\right]_{n n}\right|^{2}}{\left(\sigma_{N}^{2} / P_{R}\right) N_{T} \sum_{m=1}^{N_{T}}\left|\left[W_{k}(t)\right]_{n m}\right|^{2}+\sum_{m=1, m \neq n}^{N_{T}}\left|\left[W_{k}(t) H_{k}(t)\right]_{n m}\right|^{2}},
$$

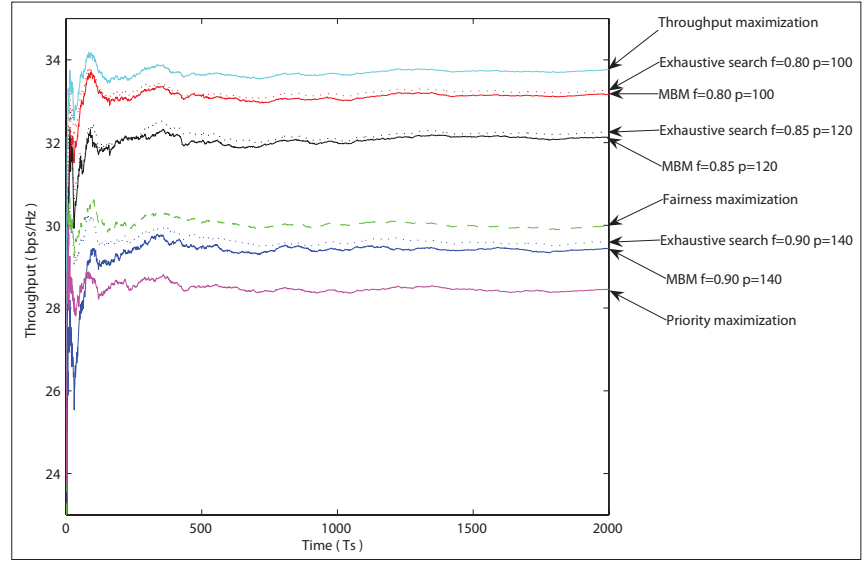

Fig. 4. Throughput comparisons for $K=N_{T}$.

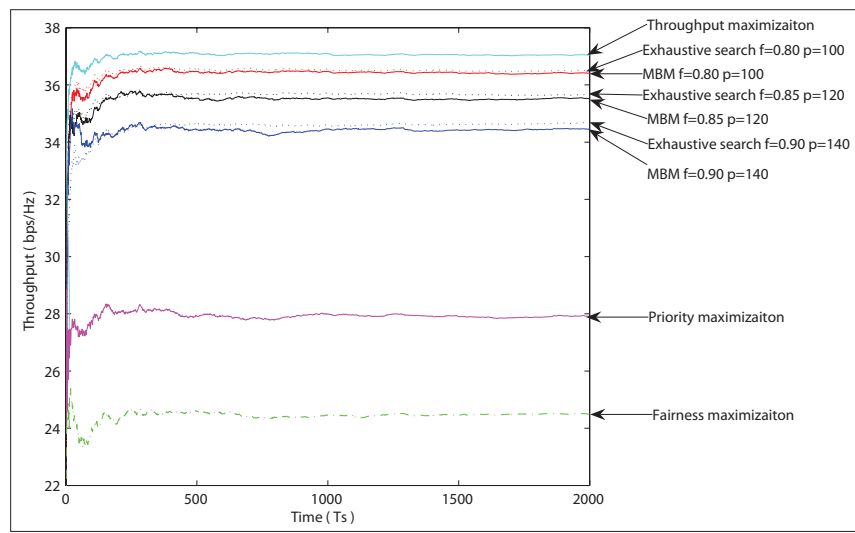

Fig. 5. Throughput comparisons for $K>N_{T}$.

We fix the number of antennas to 4 , and vary the number of active users from 3 to 5 for symmetric and asymmetric matchings. Fig. 4 shows the throughput performance comparisons for the symmetric bipartite matching case. For the generated network scenario, the Kuhn-Munkres algorithm could be applied to maximize the system capacity and the total priority. For the throughput maximization, the simulation results show the system capacity is around $33.7 \mathrm{bps} / \mathrm{Hz}$, while the long-term fairness index is around 0.79 and the total priority is around 129. In this subsection, all values of the throughput, total priority and long-term fairness index are the average values obtained over the whole simulation period. For priority maximization, the total priority is over 190, while the fairness index is lower than 0.77 , and the system capacity is just around $28.5 \mathrm{bps} / \mathrm{Hz}$. If we assume the system fairness optimization target is to have a fairness index above 0.8, the Kuhn-Munkres algorithm is inadequate. By exhaustive search, the pure fairness maximization scheme gives the best long-term fairness index over 0.9 , but it sacrifices the system capacity and priority. MBM can optimize the throughput for the given constraints of the long-term

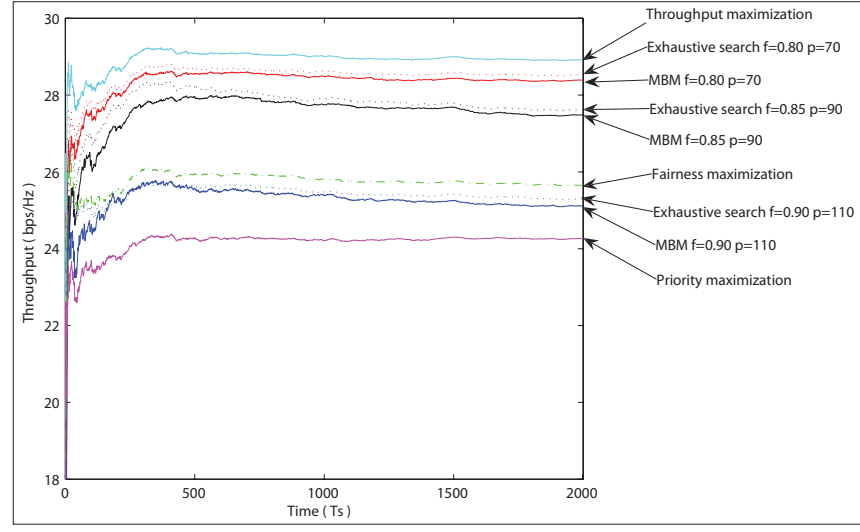

Fig. 6. Throughput comparisons for $K<N_{T}$ (1).

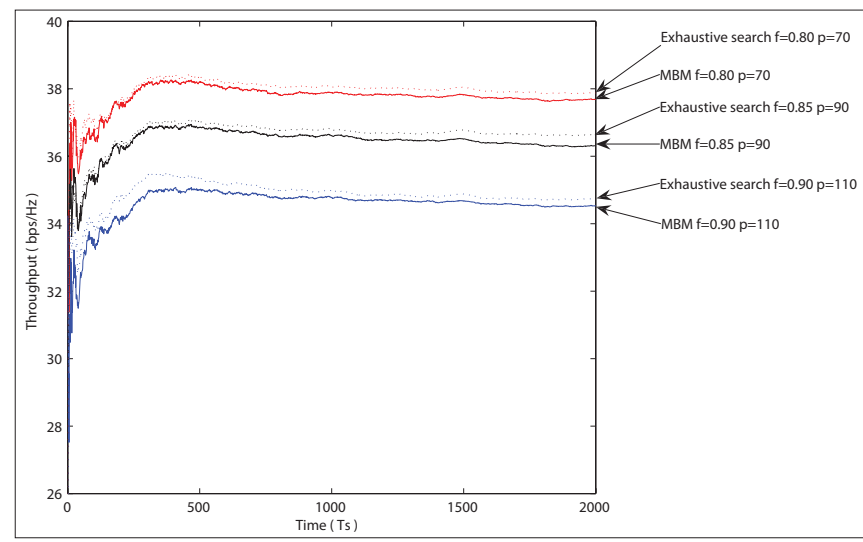

Fig. 7. Throughput comparisons for $K<N_{T}$ (2).

fairness index and priority, denoted as $f$ and $p$ in the figures. We show the MBM throughputs for different fairness index thresholds $f=0.8,0.85$, and 0.90 , and different priority thresholds $p=100,120$, and 140. For the same fairness index and priority constraints, We also give the best throughput performance under different constraints by exhaustive search from the whole matching set, as shown by the dotted lines in the Fig. 4. It is observed that our MBM solutions approach the optimal solutions.

For the asymmetric bipartite matching, Fig. 5 shows the throughput performance comparisons when $K=5(K>$ $N_{T}$ ). The result is similar to the symmetric case. Fig. 6 and Fig. 7 show the throughput performance when $K=3(K<$ $N_{T}$ ). In Fig. 6, we do not consider the utilization of the redundant antenna resource. Since only 3 users are involved in the performance evaluation of the total priority, we change the value of $p$ to 70,90 and 110. The performance of MBM is also very close to that of the exhaustive search with the same fairness index and priority constraints. In Fig. 7, if $N_{T}=4$ and $K=3$, as illustrated in Fig. 2 for asymmetric cases, we consider to reuse the remaining $N_{T}-K=1$ antenna resource, 
and assign one user with two antennas in the MIMO system, the capacity achieved for that user is greatly increased. Therefore, the system throughput performance is also improved and is much higher than the throughput performance shown in Fig. 6 when only $\min \left(N_{T}, K\right)=3$ antennas are used.

In MBM, the deletion operations try to gradually increase the performance of the sub-key objectives, while maximizing the performance of the key objective for each updated $G^{\prime}$. MBM is a type of heuristics. However, by the deletion operation, MBM tries to keep the more crucial matchings, and balance the performance of the multiple objectives. MBM fixes the size of the optimal solution set to $n^{2}$ with a low computational complexity. It is a trade-off between computational complexity and optimization performance.

\section{Conclusions}

In this paper, the multi-objective optimization problem for bipartite matchings is formulated. To give an efficient solution of this problem, based on the Kuhn-Munkres algorithm, a modified bipartite matching (MBM) algorithm is proposed. We illustrate the application of MBM to antenna assignments in wireless MIMO systems for both symmetric and asymmetric scenarios. The simulation results show that MBM can effectively reduce the matching set and dynamically provide the maximum values of the key objective, while satisfying the requirements of the other sub-key objectives.

\section{REFERENCES}

[1] H. W. Kuhn, "The Hungarian method for the assignment problem," Naval Research Logistic Quarterly, vol. 2, pp. 83-97, 1955.

[2] J. Munkres, "Algorithms for the assignment and transportation problems," J. Soc. Indust. Appl. Math., vol. 5, no. 1, Mar. 1957.

[3] K. J. Runser, J. M. Gorce, and S. Ubeda, "QoS constrained wireless LAN optimization within a multiobjective framework," IEEE Wireless Commun., vol. 13, no. 6, pp. 26-33, Dec. 2006.

[4] F. D. Rango, F. Guerriero, S. Marano, and E. Bruno, "A multiobjective approach for energy consumption and link stability issues in ad hoc networks," IEEE Commun. Lett., vol. 10, no. 1, pp. 28-30, Jan. 2006.

[5] M. Elmusrati, R. Jantti, and H. N. Koivo, "Multiobjective distributed power control algorithm for CDMA wireless communication systems," IEEE Trans. Veh. Technol., vol. 56, no. 2, pp. 778-788, Mar. 2007.

[6] R. W. Heath, M. Airy, and A. I. Paulraj, "Multiuser diversity for MIMO wireless systems with linear receivers," in Proc. Asilomor Conf. Signals, Systems, and Computers, pp. 1194-1199, Pacific Grove, CA, USA, Nov. 2001.

[7] O. S. Shin and K. B. Lee, "Antenna-assisted round Robin scheduling for MIMO cellular system," IEEE Commun. Lett., vol. 7, no. 3, pp. 109-111, Mar. 2003.

[8] Y. J. Choi, J. Kim, and S. Bahk, "Downlink scheduling with fairness and optimal antenna assignment for MIMO cellular systems," in Proc. IEEE GLOBECOM, vol. 5, pp. 3165-3169, Dallas, TX, USA, Nov. 2004.

[9] M. Torabzadeh and Y. Ji, "Efficient assignment of transmit antennas for wireless communications," in Proc. IEEE International Conference in Central Asia, pp. 1-5, Tashkent, Uzbekistan, Sept. 2006.

[10] D. Nam and C. H. Park, "Multiobjective simulated annealing: a comparative study to evolutionary algorithms," International J. Fuzzy Systems, vol. 2, no. 2, pp. 87-97, 2000.

[11] D. Mitra, F. Romeo, and A. Sangiovanni-Vincentelli, "Convergence and finite-time behavior of simulated annealing," Advanced Applied Probability, vol. 18, pp. 747-771, 1986.

[12] K. Deb, A. Pratap, S. Agarwal, and T. Meyarivan, "A fast and elitist multi-objective genetic algorithm: NSGA-II," IEEE Trans. Evolutionary Computation, vol. 6, no. 2, pp. 182-197, 2002.
[13] M. T. Jensen, "Reducing the run-time complexity of multiobjective EAs: the NSGA-II and other algorithms," IEEE Trans. Evolutionary Computation," vol. 7, no. 5, pp. 503-515, 2003.

[14] R. Jain, A. Durresi, and G. Babic, "Throughput fairness index: an explanation," ATM Forum Document Number: ATM Forum/990045, Feb. 1999.

[15] D. B. West, Introduction to Graph Theory. Prentice Hall, 2001.

[16] V. Chankong and Y. Haimes, Multiobjective Decision making Theory and methodology. New York: Elsevier Science, 1983.

[17] M. Ehrgott and S. Ruzika, "An improved $\varepsilon$-constraint method for multiobjective programming," report in Wirtschaftsmathematik http://kluedo.ub.uni-kl.de/volltexte/2005/1893/, Department of Mathematics, Technical University of Kaiserslautern, 2005.

[18] R. G. Cheng, C. J. Chang, C. Y. Shih, and Y. S. Chen, "A new scheme to achieve weighted fairness for WLAN supporting multimedia services," IEEE Trans. Wireless Commun., vol. 5, no. 5, pp. 1095-1102, May 2006.

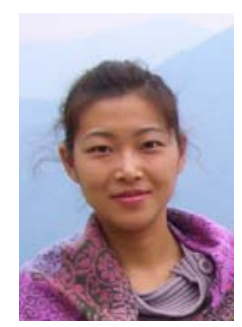

Fanglei Sun (S'06) received the BS and MS from Xidian University, Xi'an, China, in 2001 and 2004 respectively. She is currently a $\mathrm{Ph} . \mathrm{D}$. student in the Department of Electrical and Electronic Engineering, the University of Hong Kong, Hong Kong, China. She also worked as a Research Assistant from Dec. 2002 to Dec. 2003 in the same department. From Nov. 2005 to Aug. 2006, she was a Visiting Researcher in the Department of Electrical Engineering, University of California, Riverside, CA USA. Her research interests include wireless protocol design, cross-layer algorithm design over WLAN/WiMAX networks and OFDM/MIMO systems.

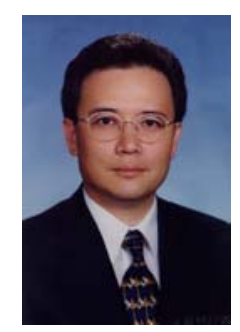

Victor O.K. Li was born in Hong Kong in 1954. $\mathrm{He}$ received SB, SM, EE and ScD degrees in Electrical Engineering and Computer Science from the Massachusetts Institute of Technology, Cambridge, Massachusetts, in 1977, 1979, 1980, and 1981, respectively. He joined the University of Southern California (USC), Los Angeles, California, USA in February 1981, and became Professor of Electrical Engineering and Director of the USC Communication Sciences Institute. Since September 1997 he has been with the University of Hong Kong, Hong Kong, where he is Associate Dean (Research) of Engineering, and Chair Professor of Information Engineering. He also served as Managing Director of Versitech Ltd. (http://www.versitech.com.hk/), the technology transfer and commercial arm of the University, from September 1997 to June 2004. Prof. Li serves on various corporate boards. His research is in information technology, including all-optical networks, wireless networks, and Internet technologies and applications. Sought by government, industry, and academic organizations, he has lectured and consulted extensively around the world. Prof. Li is very active in the research community, and has chaired various international conferences and served on the editorial boards of various international journals. He has given distinguished lectures at various universities around the world, and keynote speeches at many international conferences. Prof. Li has received numerous awards, including, most recently, the PRC Ministry of Education Changjiang Chair Professorship at Tsinghua University, Beijing, the UK Royal Academy of Engineering Senior Visiting Fellowship in Communications, the KC Wong Education Foundation Lectureship, the Croucher Foundation Senior Research Fellowship, and the Order of the Bronze Bauhinia Star, Government of the Hong Kong Special Administrative Region, China. He was elected an IEEE Fellow in 1992.

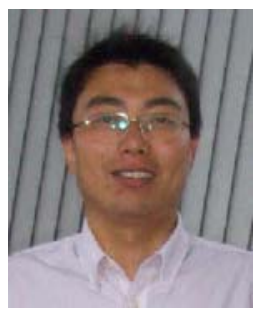

Zhifeng Diao (S'04) received the BS and MS from Xidian University, Xi' an, China, in 1999 and 2002 respectively, and $\mathrm{PhD}$ from the University of Hong Kong, Hong Kong, China in 2005. From September 2005 to September 2006, he was a postdoctoral fellow at the Department of Electrical Engineering, the University of California, Riverside, CA, USA. Currently, he is working with Huawei Technologies CO., LTD, Shanghai, China. His research interests include wireless scheduling algorithm design, smart antennas, cross layer design, OFDM, MC-CDMA,

Wimax. 